\section{FUNCTIONAL DEFECTS IDENTIFIED IN OUTGROWTH ENDOTHELIAL CELLS FROM DIABETIC PATIENTS COMPARED TO HEALTHY CONTROLS}

\begin{abstract}
1,2 Ahmad Hasan*, ${ }^{3}$ Ria Weston, ${ }^{3}$ Fiona L Wilkinson, 1,4 Andrew Boulton, ${ }^{3}$ Tawqeer Rashid, ${ }^{1,4}$ Frank Bowling, ${ }^{1,4} \mathrm{MY}$ vonne Alexander. ${ }^{1}$ Diabetes Research Group, University of Manchester, ${ }^{2}$ Universiti Kuala Lumpur, Malaysia; ${ }^{3}$ Translational Sciences, Healthcare Science Research Institute, Manchester Metropolitan University; ${ }^{4}$ Manchester Academic Health Science Centre; ${ }^{5}$ Department of Vascular and Endovascular Surgery, Manchester Royal Infirmary; *Presenting Author
\end{abstract}

\subsection{6/heartjnl-2016-309890.195}

Background Circulating endothelial progenitor cells (EPCs) are thought to play a pivotal role in endothelial repair. Clinical trials utilising EPCs to promote therapeutic angiogenesis are already underway Previous reports suggest a reduced EPC number and impaired functional activity in patients with type 2 diabetes mellitus. However, the actual identity of the cell type involved and the functional role played in the repair process needs to be further defined before EPCs can be successfully utilised in the clinic. In the present study, we assessed the functional capacity of circulating late out-growth endothelial progenitor cells (OECs) to further assess the contribution of the diabetic environment to diminished OEC function in the context of wound healing.

Methods and results OECs were isolated from 7 diabetic patients, presenting with ulcers ranging in size from $60 \mathrm{~mm}^{3}-$ $2500 \mathrm{~mm}^{3}$. OECs were characterised as $\mathrm{CD}_{3} 4^{+}, \mathrm{CD} 31^{+}$, vWF+, positive for AcLDL and UEA uptake and negative for the hematopoietic marker CD45 by immunohistochemistry. Migration of $\mathrm{CD}_{3} 4^{+}$OECs, evaluated by a scratch assay, demonstrated that migration is impaired in diabetic OECs compared to healthy control OECs, with $40-42 \%$ closure $v s$ $100 \%$ over $24 \mathrm{~h}$ respectively. In addition, achemotaxis transwell migration assay showed a decreased response to SDF-1 by diabetic cells vs healthy OECs. An angiogenesis tube formation assay also established a reduced capacity of the diabetic OECs to form an endothelial network as compared to healthy OECs $(\mathrm{p}<0.0001)$, measured using the closed loop perimeter, and the number of branch points $(\mathrm{p}<0.05)$. Nitrite concentrations were measured using a Griess assay, and the results showed that diabetic OECs produced less nitric oxide as compared to healthy OECs ( $\mathrm{p}<0.0005$ ).

Conclusion OECs from diabetic patients show an impaired migration and response to chemotactic agents in vitro compared to OECs isolated from healthy controls. In addition, the reduced nitric oxide bioavailability found by diabetic cells may contribute to OEC dysfunction in diabetes. Future work will focus on assessing the secretome of healthy vs diabetic OECs.

\section{STRATEGIES FOR INHIBITING ADVANCED GLYCATION ENDPRODUCT (AGE) INDUCED VASCULAR CALCIFICATION IN A SMOOTH MUSCLE CELL CULTURE MODEL}

${ }^{1}$ Gary P Sidgwick*, ${ }^{1}$ Peter Walling, ${ }^{1}$ Ambreen Shabbir, ${ }^{1}$ Ria Weston, ${ }^{2}$ Andrew Schiro, ${ }^{2}$ Ferdinand Serracino-Inglott, ${ }^{1}$ Alan M Jones, ${ }^{3}$ Meder Kamalov, ${ }^{3}$ Margaret A Brimble, ${ }^{1}$ Fiona L Wilkinson, ${ }^{1} \mathrm{M}$ Yvonne Alexander. 'Manchester Metropolitan University; ${ }^{2}$ Manchester Academic Health Science Centre; ${ }^{3}$ The University of Auckland; *Presenting Author

10.1136/heartjnl-2016-309890.196

Vascular calcification is implicated in a range of cardiovascular disease mechanisms, leading to an associated increase in morbidity and mortality. One such trigger are advanced glycation endproducts (AGEs), the tissue accumulation of which increases with age and is more prevalent in diabetic subjects due to oxidative stress and poor glycaemic control. The aim of this study was to investigate the osteogenic potential of AGEs and elucidate mechanisms of inhibiting these processes in a smooth muscle cell (SMC) culture model.

Osteogenic differentiation of SMCs was induced using $\hat{\mathrm{I}}^{2}$ glycerophosphate $\left(\hat{I}^{2}-\mathrm{GP}\right)$, carboxymethyllysine (CML), carboxyethyllysine (CEL) methylglyoxal (MGO) and glycated low density lipoprotein (gly-LDL). The cells were subsequently treated with aminoguanidine (AG), an inhibitor of AGE formation, and novel glycomimetic compounds in order to determine their anti-calcification potential in vitro using $\mathrm{qPCR}$, ELISA, Alkaline phosphatase (ALP) activity and Alizarin red staining.

Gly-LDL $(10 \mu \mathrm{g} / \mathrm{ml})$ and CML $(2.5 \mathrm{nM})$ increased the level of calcification observed compared to the $\hat{\mathrm{I}}^{2}-\mathrm{GP}(5 \mathrm{mM})$ positive control after 21 days $(\mathrm{p}<0.05)$, with gly-LDL induced calcification apparent after 14 days. Both AG $(250 \mu \mathrm{M})$ and the novel glycomimetic compounds reduced the level of mineralisation observed at 21 days compared with osteogenic treatments $(\mathrm{p}<0.05)$. CEL $(2.5 \mathrm{nM})$ and MGO $(0.1 \mathrm{mM})$ both induced calcification, however mineralization was not as extensive as with $\hat{\mathrm{I}}^{2}$-GP. When compared to the structure of CML, the side-chain of CEL contains an extra methyl group, suggesting this group impacts RAGE receptor binding. It was also shown that $\hat{\mathrm{I}}^{2}$-GP combined with increased glucose concentration induced more extensive calcification unlike low glucose levels and $\hat{\mathrm{I}}^{2}$-GP alone. ALP activity, when stimulated with $\hat{\mathrm{I}}^{2}$-GP, CML and gly-LDL was greater at day 4 than at day 7, with AG reducing ALP activity measurements at day 4. Gly-LDL increases gene expression of $\mathrm{OCN}$ at day $4 \mathrm{com}$ pared with $\hat{\mathrm{I}}^{2}-\mathrm{GP}$ and CML, however this was reduced at day 7 , corresponding with an increased expression of OPN and OPG. NOTCH-3 gene expression was also reduced at day 7 . Gene expression of OPN, OPG and NOTCH-3 were reduced at both day 4 and day 7 compared with osteogenic treatments ( $\hat{I}^{2}-\mathrm{GP}, \mathrm{CML}$ and gly-LDL).

In summary, we conclude that gly-LDL and CML are potent inducers of calcification compared with $\hat{\mathrm{I}}^{2}-\mathrm{GP}$, and that their osteogenic potential can be modulated by both AG and novel glycomimetic compounds.

\section{MIRNA-22 REGULATES VASCULAR SMOOTH MUSCLE CELL FUNCTIONS AND PREVENTS NEOINTIMA FORMATION BY TARGETING EVI-1}

Feng Yang*, Qingzhong Xiao. Queen Mary University of London; *Presenting Author

\subsection{6/heartjnl-2016-309890.197}

Background/objective We have recently reported that microRNA-22 (miR-22) regulates vascular smooth muscle cell (VSMC) differentiation from stem cells in vitro and in vivo. However, little is known about the functional involvements of miR-22 in VSMC functions and vessel injury-induced neointima formation. In the current study, we aimed to establish the causal role of miR-22 and its target genes in VSMC proliferation, migration and neointima lesion formation.

Methods and results miR-22 was significantly down-regulated in VSMCs upon various pathological stimuli, and in injured arteries. miR-22 over-expression in serum-starved VSMCs 
A

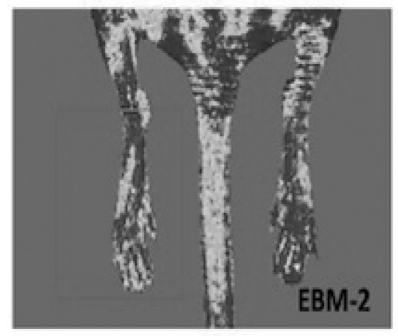

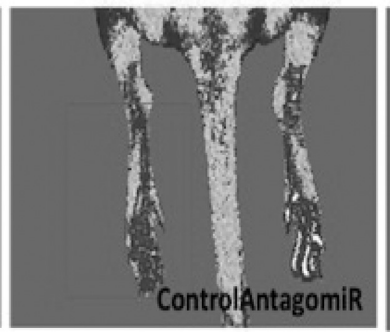
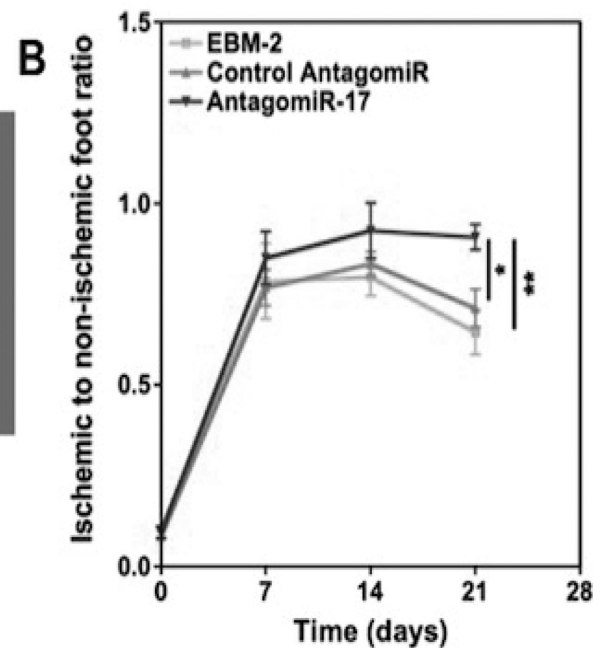

\section{Abstract 198 Figure 1}

significantly inhibited VSMC proliferation and migration, while knockdown of miR-22 dramatically promoted VSMC proliferation and migration, respectively. Ecotropic virus integration site 1 protein homolog (EVI-1), a transcription factor localised to the nucleus and binds DNA through specific conserved sequences of GACAAGATA with the potential to interact with both corepressors and coactivators, was predicted as one of the top targets of miR-22 by using several computational miRNA target prediction tools, and was negatively regulated by miR-22 in VSMCs. The luciferase activity of the wild-type, but not the miR-22 binding site mutants of EVI-1 $3 \hat{a} €^{{ }^{\mathrm{TM}}-\mathrm{UTR}}$ reporter was substantially repressed by miR-22 overexpression, confirming the EVI-1 is one of the functional targets of miR22 in VSMCs. Data from co-transfection experiments also revealed that miR-22 inhibited VSMC proliferation and migration through repressing EVI-1 gene expression. Importantly, perivascular enforced expression of miR-22 in the injured vessels significantly reduced EVI-1 expression levels, decreased VSMC proliferation, and inhibited neointima formation in wire-injured femoral arteries.

Conclusions/implications Our data have demonstrated that miR-22 is an important regulator in VSMC functions and neointima hyperplasia, suggesting its potential therapeutic application for vascular diseases.

\section{MICRORNA-17 AS THE TARGET OF IMMOBILIZED VASCULAR ENDOTHELIAL GROWTH FACTOR IN ENDOTHELIAL CELL SURVIVAL UNDER ISCHAEMIC CONDITIONS}

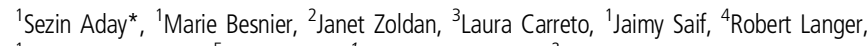
${ }^{1}$ Costanza Emanueli, ${ }^{5}$ Lino Ferreira. ${ }^{1}$ University of Bristol; ${ }^{2}$ The University of Texas at Austin; ${ }^{3}$ University of Aveiro; ${ }^{4}$ Massachusetts Institute of Technology; ${ }^{5} \mathrm{CNC}$-Center for Neuroscience and Cell Biology; *Presenting Author

\subsection{6/heartjnl-2016-309890.198}

The development of tools to control the cell activity after transplantation is of utmost importance in Regenerative Medicine. For clinical efficacy, it is imperative that cells survive and engraft into the host tissue. However, a significant number of cells (typically $>70 \%$ in few days) die or are lost within hours after transplantation. Therefore, the implementation of new strategies to promote cell survival after transplantation is crucial.

Both cord blood-derived endothelial progenitor cells (CBEPCs) and VEGF-A have been proposed as therapeutic tools to induce angiogenesis for the treatment of ischemic diseases. VEGF-A promotes the formation of microvessels that differ structurally and functionally depending on its presentation. Free VEGF forms big, leaky vessels while immobilized VEGF facilitates the formation of highly branched networks. Immobilization of VEGF alters VEGFR-2 signaling and subsequent downstream pathways. Improved understanding of the molecular targets underlying the action mechanism of immobilized VEGF might help to develop new platforms to increase posttransplantation cell survival and regenerative capacity.

We showed that conjugated VEGF modulates cord bloodderived endothelial cell (CB-EC) activity by decreasing the expression miR-17 both in vitro and in vivo. To mimic the down-regulation of miR-17 by immobilized VEGF and understand the effect of miR-17 down-regulation on cell survival, CB-ECs were transfected with antagomiR-17. AntagomiR-17 increased CB-EC survival at least 1.5 times $(n=6)$ compared to scrambled antagomiR or pro-angiogenic miRNAs reported in the literature (e.g. miR-424 and miR-132) and sprout formation on Matrigel at least 2 times $(n=5)$ under hypoxia conditions $\left(0.1 \% \quad \mathrm{O}_{2}\right)$. In vivo, antagomiR-17 accelerated hemodynamic recovery of the whole $\operatorname{limb}(n=12)$ in unilateral limb ischemia obtained by occlusion of the left femoral artery (Figure1).

Blood flow recovery evaluated by Laser Doppler analysis was significantly higher 21 days after surgery in antagomiR-17 group compared to scrambled antagomiR and EBM-2 groups. Immunohistochemical analyses showed around 1.4 times increase in the capillary density of skeletal muscle in antagomiR-17 condition. In order to determine the gene target and potential pathway involved in the biological effect of antagomiR-17, next generation mRNA sequencing and data mining were used. Gene expression results and siRNA experiments on CB-ECs revealed ZNF652 and CDKN1A as the targets of antagomiR-17-mediated EC survival. Interestingly, CDKN1A was reported in the literature as an anti-apoptotic molecule. 